\title{
Atypical presentation of tracheoesophageal fistula: immediate failure of ventilation via tracheostomy tube
}

\author{
Qi-Yi Bong*, Doh Jeing Yong, Heng Yao Tan
}

Department of Otorhinolaryngology, Queen Elizabeth Hospital, Kota Kinabalu, Sabah, Malaysia

Received: 17 January 2019

Accepted: 12 March 2019

\author{
*Correspondence: \\ Dr. Qi-Yi Bong, \\ E-mail: bong1123@hotmail.com
}

Copyright: () the author(s), publisher and licensee Medip Academy. This is an open-access article distributed under the terms of the Creative Commons Attribution Non-Commercial License, which permits unrestricted non-commercial use, distribution, and reproduction in any medium, provided the original work is properly cited.

\begin{abstract}
Tracheoesophageal fistula (TOF) is a rare complication of prolonged endotracheal intubation. Undiagnosed TOF during tracheostomy poses a risk of improper placement of tracheostomy tube and can lead to a potentially lifethreatening situation. We report a case of an undiagnosed tracheoesophageal fistula during tracheostomy in a lady in which we encountered difficulty in placement of tracheostomy tube and were eventually able to cannulate the trachea with the help of a fibre optic bronchoscope.
\end{abstract}

Keywords: Prolonged ventilation, Tracheostomy, Tracheoesophageal fistula, Fibre optic bronchoscope

\section{INTRODUCTION}

Acquired benign tracheoesophageal fistula (TOF) in adult is a rare condition. It has been reported due to postintubation injuries, blunt and penetrating trauma, granulomatous mediastinal infections, prior oesophageal surgery, prior tracheal or laryngeal surgery, iatrogenic injuries and indwelling stents. ${ }^{1}$ The incidence of TOF due to prolonged duration of endotracheal intubation is between $0.3 \%$ and $3 \% .^{2}$ Although the use of high volume, low pressure cuffs has reduced the incidence of TOF, it still poses a challenge to maintain airway in patients requiring ventilator support. An undiagnosed TOF during tracheostomy can lead to entry of tube into oesophagus through fistula. Hence, TOF is an important differential to consider in failure to ventilate during tracheostomy. Fibre optic bronchoscope is an invaluable tool to aid positioning of tube distal to TOF.

\section{CASE REPORT}

A 58 year old female with underlying iron deficiency anaemia was initially admitted for septic shock secondary to left lower limb bullous haemorrhagic cellulitis. During her stay in the hospital, patient had multiple episodes of intubation for debridement surgery of her left lower limb and recurrent pneumonia for a total of 22 days. There were no difficulties during each intubation; all were single attempt using cuffed $7.0 \mathrm{~mm}$ internal diameter (ID) endotracheal tube (ETT). She was also on Ryle's tube feeding for prolonged period. Patient was then referred to us for tracheostomy for prolonged ventilation.

Surgical tracheostomy was carried out in the operation theatre and no difficulties were encountered until insertion of tracheostomy tube. Tracheotomy performed no obvious abnormalities of trachea were found. Tracheal dilator was inserted into tracheostoma and endotracheal tube was retracted up under direct visualization. Cuffed $7.5 \mathrm{~mm}$ ID tracheostomy tube size was then inserted. Immediately after insertion, there were no waveforms seen on the capnography and failure to ventilate. False tract was suspected. Thus, tracheostomy tube was removed and ETT was re-advanced to ventilate the patient. Insertion of tracheostomy tube was reattempted with similar outcome. Consecutive confirmation by anaesthesiologist using fibre optic bronchoscope through the ETT which was located proximal to the tracheostoma 
confirmed that the shaft of tracheostomy tube is located in the tracheal lumen. However, failure to ventilate persisted. Fibre optic bronchoscope through tracheostomy tube revealed that the distal part of tracheostomy tube was not located in the tracheal lumen. Tracheostomy tube was then removed and ETT re-advanced again. However, this resulted in failure to ventilate which lead to asystole and cardiopulmonary resuscitation was commenced. Otorhinolaryngology surgeon noted tip of ETT was outside of the tracheal lumen using a fibre optic bronchoscope through the ETT. ETT was gently withdrawn and advanced into tracheal lumen under fibre optic bronchoscope. Patient achieved return of spontaneous circulation after 20 minutes and was then admitted to intensive care unit due to unstable haemodynamic status.

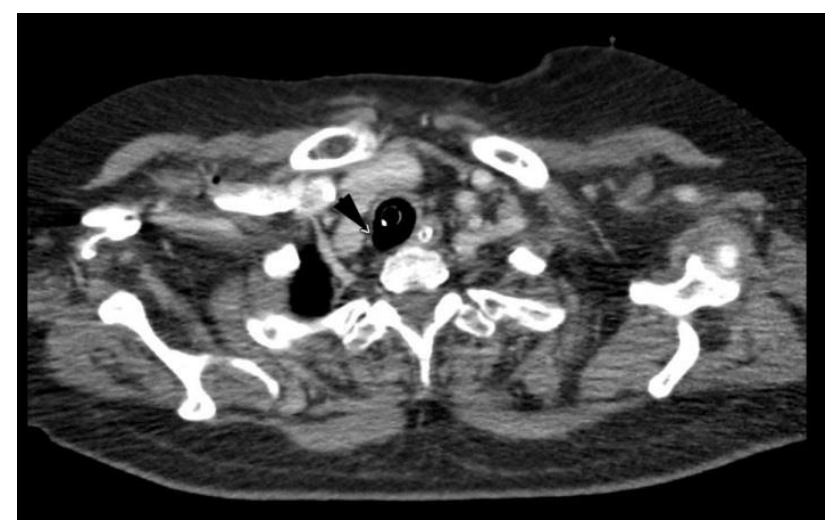

Figure 1: Thinning and discontinuation of the right posterolateral tracheal wall (black arrow).

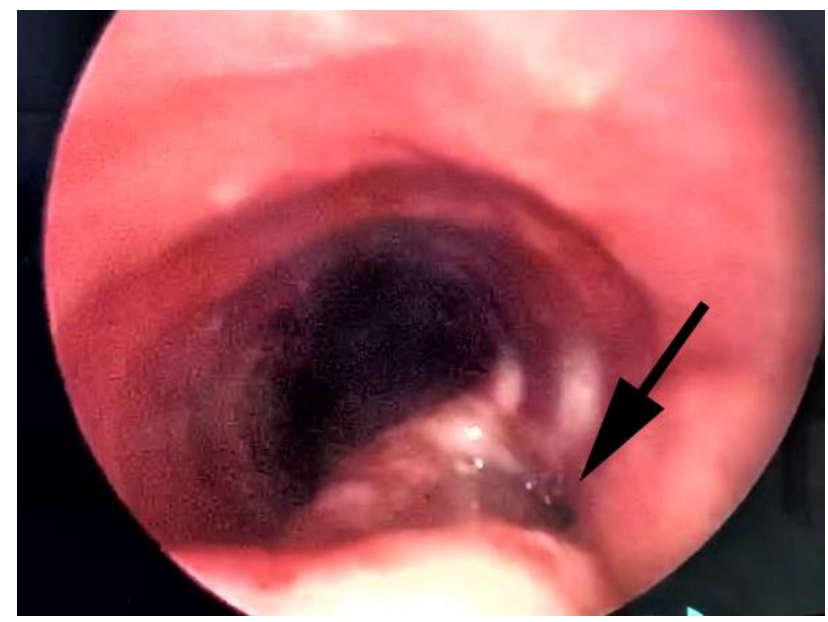

Figure 2: Tracheoesophageal fistula (black arrow).

Computed tomography of thorax revealed thinning and discontinuity of the right posterolateral tracheal wall at T2 vertebrae level (Figure 1). Examination under anaesthesia, neck exploration, tracheostomy and airway study was performed. Intraoperatively, a tracheoesophageal fistula located $3 \mathrm{~cm}$ distal to vocal cords and measuring $3 \mathrm{~cm}$ in length was noted (Figure 2). As a standard sized tracheostomy tube was unable to bypass the fistula, a size $8.0 \mathrm{~mm}$ ID extended-length tracheostomy tube was inserted under fibre optic bronchoscopy guidance.

Corrective surgery was planned for another date after weaning off positive pressure ventilation.

\section{DISCUSSION}

TOF can develop after a prolonged duration of intubation ranging from 12 to 200 days with a mean of 40 days. $^{3}$ The inflated endotracheal tube cuff can cause ischaemic injury to the tracheal mucosa, and combine with impingement from an indwelling nasogastric tube leads to pressure necrosis between trachea and oesophagus. Other factors implicated in development of TOF are high cuff pressure, high airway pressure, excessive motion of tracheal tube, respiratory infections, oesophageal infections, hypotension, and advanced age. ${ }^{4}$

Clinical manifestation of TOF differs according to patient's respiratory status; patients on mechanical ventilation may present with air leaks despite hyperinflated cuff, abdominal distension, tracheobronchial contamination with food and digestive secretions and bronchopulmonary suppuration; patients with normal breathing may present with aspiration symptoms and bronchopulmonary suppuration with respiratory deterioration. ${ }^{5}$ Some symptoms are nonspecific, hence, diagnosis is not made prior to tracheostomy as in our case.

When failure to ventilate upon insertion of tracheostomy tube is encountered, the possibility of TOF should be considered in addition to false tract and tracheal transection.

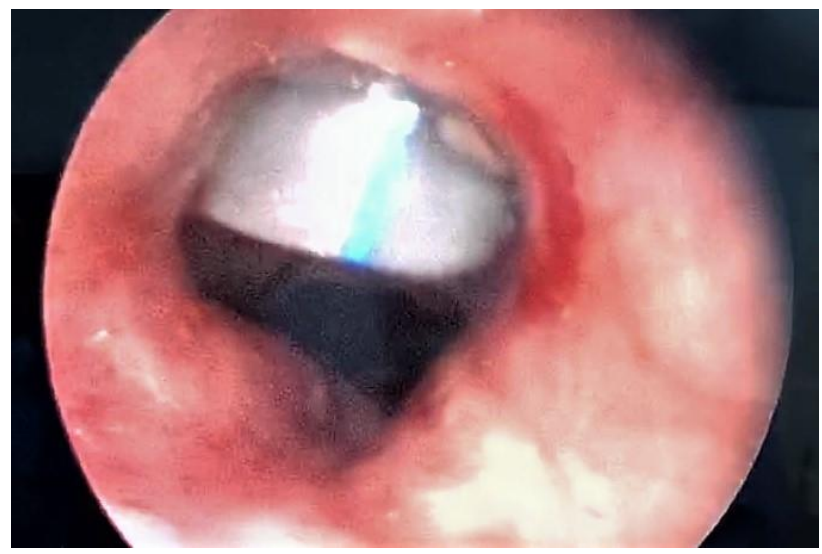

Figure 3: Tracheostomy tube inserted directly into tracheoesophageal fistula.

In our case of undiagnosed TOF, tracheostomy tube has entered the oesophagus through tracheostoma and TOF upon insertion (Figure 3). The usage of fibre optic bronchoscope through tracheostomy tube is able to confirm the location of tube and aid in manoeuvring the 
tube into tracheal lumen instead of through the TOF. We suggest that fibre optic bronchoscope through tracheostomy tube is used to confirm its location after the first attempt of failure to ventilate post insertion of tracheostomy tube. This reduces the mortality due to failure to ventilate.

\section{CONCLUSION}

TOF is a rare complication of prolonged duration of intubation. High index of suspicion for TOF should be maintained when failure to ventilate upon insertion of tracheostomy tube. Usage of fibre optic bronchoscope through tracheostomy tube is invaluable in cases of TOF to ensure proper placement of tube.

Funding: No funding sources Conflict of interest: None declared

Ethical approval: Not required

\section{REFERENCES}

1. Shen KR, Allen MS, Cassivi SD, Nichols FC. III, Wigle DA, Harmsen WS, et al. Surgical management of acquired nonmalignant tracheoesophageal and bronchoesophageal fistulae. Ann Thorac Surg. 2010;90:914-8.

2. Couraud L, Ballester MJ, Delaisement C. Acquired tracheoesophageal fistula and its management. Semin Thorac Cardiovasc Surg. 1996;8:392-9.

3. Marzelle J, Dartevelle P, Khalife J, Rojas-Miranda A, Chapelier A, Levasseur P. Surgical management of acquired postintubation TOF. Eur J Cardiothorac Surg. 1989;3:499-503.

4. Kastanos N, Estopa Miro R, Marin Perez A, Xaublet Mir A, Agusti-Vidal A. Laryngotracheal injury due to endotracheal intubation: Incidence, evolution, and predisposing factors.A long term study. Crit Care Med. 1983;11:362-7.

5. Paraschiv M. Tracheoesophageal fistula: A complication of prolonged tracheal intubation. J Med Life. 2014;7:516-21.

Cite this article as: Bong QY, Yong DJ, Tan HY. Atypical presentation of tracheoesophageal fistula: immediate failure of ventilation via tracheostomy tube. Int J Otorhinolaryngol Head Neck Surg 2019;5:769-71. 